\title{
Protozoa hosts lead to virulence
}

\author{
The combined selective pressures of residing in different protozoan hosts in the environment drives the evolution of \\ virulence in the opportunistic pathogen Legionella pneumophila.
}

\section{Diane McDougald and Sharon R. Longford}

C onventional understanding proposes that interactions of pathogenic bacteria with human hosts result in selection for traits that increase virulence or survival in the host. But for opportunistic pathogens that are acquired from the environment, these traits must have evolved for increased fitness of the pathogen in the environment, as most of these bacteria are not transmitted from person to person. One such pathogen is Legionella pneumophila, which is ubiquitous in freshwater systems and replicates inside single-celled protozoa, such as amoeba ${ }^{1}$.

Within the human host, the $L$. pneumophila pathogen is engulfed via phagocytosis into macrophage cells where it replicates ${ }^{2}$. This Legionella-containing vacuole (LCV) requires the Dot/Icm type IV secretion system to transport more than 300 effector molecules from the pathogen into the host cell ${ }^{3}$. While the Dot/Icm system is required for L. pneumophila survival in the macrophage, the deletion of individual Icm/ Dot translocated substrates (IDTSs) rarely affects intracellular growth and survival. Thus, many of the effectors are redundant, and deletion of individual genes does not necessarily affect the pathogen's intracellular survival or ability to replicate in macrophages.

In Park et al. ${ }^{5}$, the authors hypothesize that L. pneumophila's ability to replicate in human macrophages is due to the evolution of genes that enable growth inside amoebae in the environment. In fact, L. pneumophila grows within a broad range of protozoan hosts $^{4}$. Therefore, it is likely that different genes are required for replication in the different protozoan hosts, and this might account for many of the redundant genes in macrophages ${ }^{5}$.

The authors used a transposon sequencing screening strategy to identify L. pneumophila genes required for growth in four diverse amoebal hosts: Acanthamoeba castellanii and Acanthamoeba polyphaga, Hartmannella vermiformis from a different class, and Naegleria gruber from a different phylum. They show that indeed different

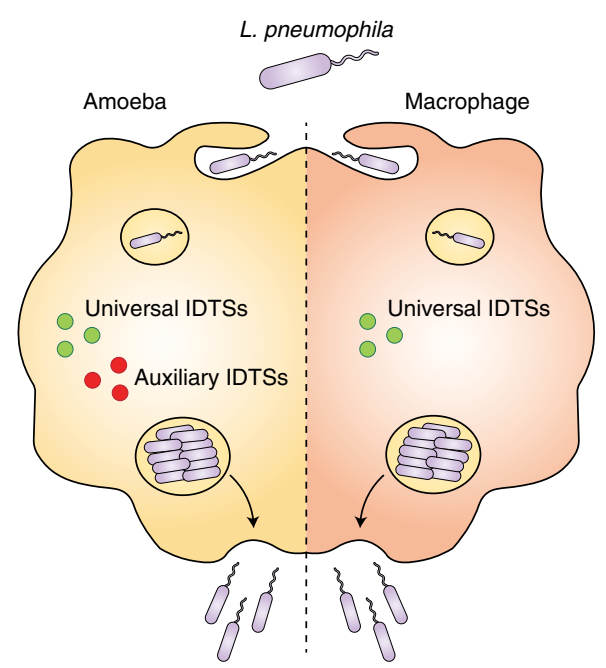

Fig. 1 Similarities between the intracellular environment of amoeba and macrophages during L. pneumophila invasion. Growth of L. pneumophila in multiple amoeba species leads to selection for various virulence genes that also promote bacterial growth in human macrophages. Universal individual IDTSs that are important for growth in all amoebal hosts are also required for replication in human macrophages, while the majority of auxiliary IDTSs, important in one or more amoebae, are not

sets of genes are important in the different hosts, with 253, 239, 225 and 186 genes being important for L. pneumophila growth in A. castellanii, A. polyphaga, $H$. vermiformis and $N$. gruberi, respectively, and only 87 of these genes (18 of which are IDTS genes) are required for growth in all the hosts tested. Of all the genes important for growth in at least one amoeba, 44 were IDTS genes.

Testing of mutants in 22 of the 44 IDTS genes for growth in amoeba revealed a subset of IDTS genes important in all amoeba (universal IDTSs) and a subset important in one or more of the amoebae (auxiliary IDTSs). In particular, mutations in three specific genes ( $s d h A, \operatorname{rav} Y$ and lpg1751) affected growth in all four amoebae, and these genes were also required for growth in macrophages. All of the universal IDTSs were required for growth in human macrophages, while all but one of the auxiliary IDTSs were not. This indicates that they have traits or targets that are similar in amoebae and macrophages. Similarly, the biotin and thiamine biosynthetic pathways were required for L. pneumophila growth in amoebae and for replication in macrophages.

The work by Park et al. ${ }^{5}$ supports the concept of convergent evolution of virulence, whereby growth in multiple amoeba species led to selection for various virulence genes that also allowed L. pneumophila to grow in human macrophages. This further supports the view that interactions with protozoa in the environment are a driving force for evolution of virulence in opportunistic pathogens and that infections in human hosts may be an accidental consequence of these bacterial-protozoa interactions $^{6}$ (Fig. 1).

\section{Diane McDougald (D) 1,2凶 and} Sharon R. Longford ${ }^{2}$

${ }^{1}$ The ithree Institute, University of Technology Sydney, Sydney, New South Wales, Australia. ${ }^{2}$ Singapore Centre for Environmental Life Sciences Engineering, Nanyang Technological University, Singapore, Singapore.

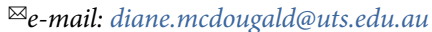

Published online: 26 March 2020

https://doi.org/10.1038/s41564-020-0699-8

References

1. Albert-Weissenberger, C., Cazalet, C. \& Buchrieser, C. Cell Mol. Life Sci. 64, 432-448 (2007).

2. Horwitz, M. A. J. Exp. Med. 158, 1319-1331 (1983).

3. Vogel, J. P., Andrews, H. L., Wong, S. K. \& Isberg, R. R. Science 279, 873-876 (1998)

4. Boamah, D. K., Zhou, G., Ensminger, A. W. \& O'Connor, T. J. Front. Cell. Infect. Microbiol. 7, 477 (2017).

5. Park, J. M., Ghosh, S. \& O’Connor, T. J. Nat. Microbiol. https://doi. org/10.1038/s41564-019-0663-7 (2020)

6. Erken, M., Lutz, C. \& McDougald, D. Microb. Ecol. 65, 860-868 (2013).

Competing interests

The authors declare no competing interests. 